\title{
THE PRACTICAL COMPUTATION OF AREAS ASSOCIATED WITH BINARY QUARTIC FORMS
}

\author{
MICHAEL A. BEAN
}

\begin{abstract}
We derive formulas for practically computing the area of the region $|F(x, y)| \leq 1$ defined by a binary quartic form $F(X, Y) \in \mathbb{R}[X, Y]$. These formulas, which involve a particular hypergeometric function, are useful when estimating the number of lattice points in certain regions of the type $|F(x, y)| \leq$ $h$ and will likely find application in many contexts. We also show that for forms $F$ of arbitrary degree, the maximal size of the area of the region $|F(x, y)| \leq 1$, normalized with respect to the discriminant of $F$ and taken with respect to the number of conjugate pairs of $F(x, 1)$, increases as the number of conjugate pairs decreases; and we give explicit numerical values for these normalized maxima when $F$ is a quartic form.
\end{abstract}

\section{INTRODUCTION}

Let $F(X, Y)=a_{0} X^{n}+a_{1} X^{n-1} Y+\cdots+a_{n} Y^{n}$ be a binary form with real coefficients and let $A_{F}$ denote the area of the region $|F(x, y)| \leq 1$. (That $|F(x, y)| \leq$ 1 defines a region with area is clear from the polar form $r \leq|F(\cos \theta, \sin \theta)|^{-1 / n}$ of this inequality.) Let $D_{F}$ denote the discriminant of $F$. If $F$ has the factorization $\prod_{i=1}^{n}\left(\alpha_{i} X-\beta_{i} Y\right)$ with $\alpha_{i}, \beta_{i} \in \mathbb{C}$ (every binary form has such a factorization), then $D_{F}=\prod_{i<j}\left(\alpha_{i} \beta_{j}-\alpha_{j} \beta_{i}\right)^{2}$. Let $B(x, y)$ denote the Beta function with arguments $x$ and $y$.

In [2], we showed that if $F$ has degree $n \geq 4$ and discriminant $D_{F} \neq 0$, then

$$
\left|D_{F}\right|^{1 / n(n-1)} A_{F} \leq 2^{7 / 6} B\left(\frac{1}{4}, \frac{1}{2}\right) \approx 11.77
$$

moreover, we showed that the bound $2^{7 / 6} B(1 / 4,1 / 2)$ is attained, for example, when $F(X, Y)=X Y\left(X^{2}-Y^{2}\right)$. This result improved an earlier estimate for $A_{F}$ given in [3]. There we showed that if $F$ has degree $n \geq 3$, then $\left|D_{F}\right|^{1 / n(n-1)} A_{F} \leq$ $3 B(1 / 3,1 / 3) \approx 15.90$, with equality holding when $F(X, Y)=X Y(X-Y)$; in fact, we showed that the sequence $\left\{M_{n}\right\}$ defined by $M_{n}=\max \left|D_{F}\right|^{1 / n(n-1)} A_{F}$ (the maximum being taken with respect to forms of degree $n$ ) is a decreasing sequence, but we did not determine the values of $M_{n}$ for $n \geq 4$. ${ }^{1}$

Received by the editor August 2, 1994 and, in revised form, February 14, 1996.

1991 Mathematics Subject Classification. Primary 11D75, 51M25; Secondary 11-04, 11E76, 11H06, 33C05, 51M16.

Key words and phrases. Elliptic integral, hypergeometric function, Thue inequality.

${ }^{1}$ The results quoted in this paragraph actually hold in the more general context of forms with complex coefficients; the reason for this is that the quantity $\left|D_{F}\right|^{1 / n(n-1)} A_{F}$, when considered over the class of forms of degree $n$ with complex coefficients, is always maximized by a form with real coefficients (see [3]). However, many of the results stated in subsequent paragraphs do not hold in this more general setting; consequently, the reader should assume throughout that $F(X, Y) \in \mathbb{R}[X, Y]$ unless otherwise indicated. 
The quantity $\left|D_{F}\right|^{1 / n(n-1)} A_{F}$ actually has only two possible values when $F$ has degree three or two. To be precise, if $F$ has degree three, then

$$
\left|D_{F}\right|^{1 / 6} A_{F}=\left\{\begin{array}{cl}
3 B\left(\frac{1}{3}, \frac{1}{3}\right) & \text { if } D_{F}>0, \\
\sqrt{3} B\left(\frac{1}{3}, \frac{1}{3}\right) & \text { if } D_{F}<0
\end{array}\right.
$$

while if $F$ has degree two

$$
\left|D_{F}\right|^{1 / 2} A_{F}= \begin{cases}\infty & \text { if } D_{F}>0 \\ 2 \pi & \text { if } D_{F}<0\end{cases}
$$

(See $\S 6$ of $[3]$ for the derivation of $\left|D_{F}\right|^{1 / 6} A_{F}$ when $D_{F}>0$; the other derivations are entirely analogous.) This stands in marked contrast to the situation in general. Indeed, if $n \geq 4$, then $\left|D_{F}\right|^{1 / n(n-1)} A_{F}$ assumes all real values between 0 and $M_{n}$ as $F$ runs over the forms of degree $n$ (see [5]).

Equations (2) and (3) enable us to calculate the value of $A_{F}$ in a direct and elementary manner when $F$ is a cubic or a quadratic form with non-zero discriminant. It is natural to ask whether there are elementary formulas (necessarily dependent on quantities besides $D_{F}$ ) which give the exact value of $A_{F}$ in general. Such formulas would likely find application in many contexts since the regions $|F(x, y)| \leq h$ are among the most basic and natural that one could consider in two dimensions.

Our primary motivation for seeking formulas for $A_{F}$ actually arises from a particular theorem of Mahler concerning lattice points in certain regions of the type $|F(x, y)| \leq h$. Mahler [8] showed that if $F$ has integer coefficients, degree $n \geq 3$, and is irreducible over $\mathbb{Q}$, then the number $N_{F}(h)$ of lattice points in the region $|F(x, y)| \leq h$ and the area $A_{F} h^{2 / n}$ of this region are connected by the relationship

$$
\left|N_{F}(h)-A_{F} h^{2 / n}\right| \leq c_{F} h^{1 /(n-1)}
$$

where $c_{F}$ is a number depending only on $F .^{2}$ From this relationship, it is clear that a general formula for $A_{F}$ would be very useful in the study of $N_{F}(h)$.

In this paper, we will derive formulas for calculating $A_{F}$ when $F$ is a quartic form. The formulas which we give will involve a particular hypergeometric function and will be practical for computation. Our analysis will divide into three cases according to the number of pairs of complex conjugate roots possessed by $F(x, 1)$. For quartic forms with real coefficients, the number of such pairs is zero, one, or two.

This division of cases is quite natural to consider since the number of pairs of complex conjugate roots of $F(x, 1)$ determines (and is determined by) the number of singularities in the polar integral representation of $A_{F}$. To be precise, if $F(x, 1)$ has $k$ pairs of complex conjugate roots, then the graph of $|F(x, y)|=1$ (in the real affine plane) has $n-2 k$ asymptotes (each one arising from a different real linear

\footnotetext{
${ }^{2}$ Mahler's result does not hold for all forms with real coefficients; indeed, if even one of the roots of $F(x, 1)$ is a Liouville number, then $N_{F}(h)$ is infinite while $A_{F}$ remains finite. On the other hand, the stated conditions are stronger than necessary to ensure the finiteness of $N_{F}(h)$ and can likely be relaxed.
} 

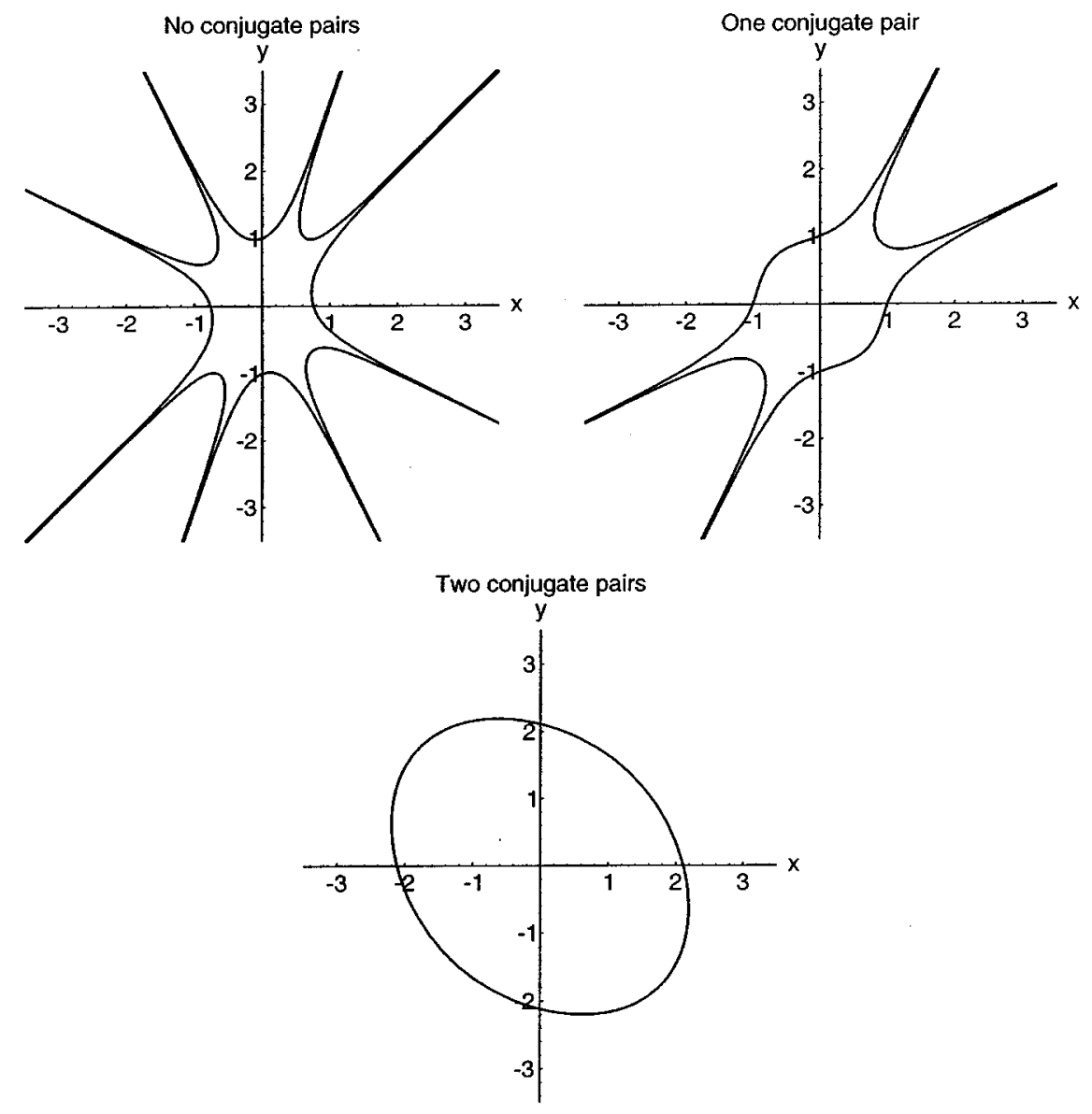

FiguRE 1. Sample graphs for the three types of quartics

factor of $F(X, Y))$, and so the calculation of $A_{F}$ involves the treatment of $2(n-2 k)$ singularities. ${ }^{3}$ Figure 1 provides an illustration of these cases when $n=4$.

A similar division of cases was implicitly used in the derivation of equations (2) and (3); indeed, for cubic and quadratic forms $F$, the sign of $D_{F}$ indicates the presence (or absence) of a conjugate pair. In general, we should expect the calculation of $A_{F}$ for forms of degree $n$ to involve $\left[\frac{n}{2}\right]+1$ cases (as determined by the number of conjugate pairs of $F(x, 1)$ ). Hence, the task of finding elementary formulas for forms of higher degree could become increasingly more complicated.

Although we are unable to derive formulas for $A_{F}$ in all ${ }^{4}$ the $\left[\frac{n}{2}\right]+1$ cases described above, we can still prove an important relationship among these cases. In particular, we will show that for forms of degree $n$, the maximal value of $\left|D_{F}\right|^{1 / n(n-1)} A_{F}$ taken with respect to the number of conjugate pairs decreases as the number of conjugate pairs increases. A brief glance at equations (2) and (3)

\footnotetext{
${ }^{3}$ Since $F$ has real coefficients, the number of asymptotes of $|F(x, y)|=1$ must be $n-2 k$ for some $k$ (the number of pairs of complex conjugate roots). For forms with complex coefficients, this need not be true since the non-real roots of $F(x, 1)$ need not come in conjugate pairs.

${ }^{4}$ Formulas for $A_{F}$ when $F$ has a complete factorization over $\mathbb{R}$ were given in [2].
} 
indicates that this result is clearly true for cubics and quadratics; however, its truth in general is not at all obvious since $\left|D_{F}\right|^{1 / n(n-1)} A_{F}$ assumes all real values between 0 and $M_{n}$. We will prove this result by appealing to the maximum principle for plurisubharmonic functions from the theory of several complex variables.

With this general result in hand, we will then return to the special class of quartic forms to compute explicit numerical values for the maxima of $\left|D_{F}\right|^{1 / n(n-1)} A_{F}$ (in each of the three cases determined by the number of conjugate pairs of $F$ ). We will conclude with two minor results which show that the computed maxima are in fact the true maxima.

\section{Statement of Results}

Let $F(X, Y)=a_{0} X^{4}+a_{1} X^{3} Y+a_{2} X^{2} Y^{2}+a_{3} X Y^{3}+a_{4} Y^{4}$ be a binary quartic form with real coefficients and non-zero discriminant, and let $k$ be the number of pairs of complex conjugate roots of the polynomial $F(x, 1)$. Note that $k$ is the number of definite quadratic factors in the factorization of $F(X, Y)$ over $\mathbb{R}$ and has the value zero, one, or two.

Suppose that $F(X, Y)$ has the factorization $\prod_{i=1}^{4}\left(\alpha_{i} X-\beta_{i} Y\right)$ with the fractions $\alpha_{i} / \beta_{i}$ arranged such that

$$
\left\{\begin{array}{l}
\frac{\alpha_{1}}{\beta_{1}}<\frac{\alpha_{2}}{\beta_{2}}<\frac{\alpha_{3}}{\beta_{3}}<\frac{\alpha_{4}}{\beta_{4}} \quad \text { if } k=0 \\
\frac{\alpha_{1}}{\beta_{1}}<\frac{\alpha_{2}}{\beta_{2}}, \quad \frac{\alpha_{3}}{\beta_{3}}=\frac{\overline{\alpha_{4}}}{\overline{\beta_{4}}} \quad \text { if } k=1 \\
\frac{\alpha_{1}}{\beta_{1}}=\frac{\overline{\alpha_{2}}}{\overline{\beta_{2}}}, \quad \frac{\alpha_{3}}{\beta_{3}}=\frac{\overline{\alpha_{4}}}{\overline{\beta_{4}}} \quad \text { if } k=2
\end{array}\right.
$$

(Here $\bar{\alpha}$ denotes the complex conjugate of $\alpha$.) Put

$$
\rho_{F}=\frac{\left(\alpha_{4} \beta_{1}-\alpha_{1} \beta_{4}\right)\left(\alpha_{3} \beta_{2}-\alpha_{2} \beta_{3}\right)}{\left(\alpha_{4} \beta_{2}-\alpha_{2} \beta_{4}\right)\left(\alpha_{3} \beta_{1}-\alpha_{1} \beta_{3}\right)}
$$

and define $\sigma_{F} \in(0,1)$ by

$$
\sigma_{F}=\sigma_{F}^{(k)}= \begin{cases}\frac{\rho_{F}}{2}\left(1+\sqrt{\frac{1+\Re \rho_{F}}{2}}\right) & \text { if } k=0, \\ \left(\frac{\sqrt{\rho_{F}}-1}{\sqrt{\rho_{F}}+1}\right)^{2} & \text { if } k=2 .\end{cases}
$$

(The notation $\sigma_{F}^{(k)}$, while logically redundant, emphasizes the dependence of $\sigma_{F}$ on $k$ and will be particularly useful when considering collections of forms with a given $k$ value. Note that the arrangement of the fractions $\alpha_{i} / \beta_{i}$ assumed in (4) is not uniquely determined by $F$; in particular, this arrangement is not invariant under complex conjugation. However, all arrangements of type (4) associated with 
a specific $F$ give rise to the same value of $\sigma_{F} ;{ }^{5}$ moreover, the assumptions of (4) guarantee that $\sigma_{F} \in(0,1)$.)

Let $I$ be the function

$$
I(x)=\int_{0}^{1} \frac{d z}{z^{1 / 2}(1-z)^{1 / 2}(1-x z)^{1 / 2}}, \quad x \in(0,1),
$$

and define functions $J_{0}, J_{1}, J_{2}$ by

$$
\begin{aligned}
& J_{0}(x)=2 x^{1 / 6}(1-x)^{1 / 6}(I(x)+I(1-x)), \\
& J_{1}(x)=2^{1 / 3} x^{1 / 12}(1-x)^{1 / 12}(I(x)+I(1-x)), \\
& J_{2}(x)=2^{1 / 3} x^{1 / 12}(1-x)^{1 / 3} I(1-x)
\end{aligned}
$$

for $x \in(0,1)$.

We will derive the following representation for $A_{F}$ in $\S 3$.

Theorem 1. Let $F(X, Y)$ be a binary quartic form with real coefficients and nonzero discriminant $D_{F}$, and let $k$ be the number of pairs of complex conjugate roots of the polynomial $F(x, 1)$. Then, with the notation above, we have

$$
A_{F}= \begin{cases}\frac{J_{0}\left(\sigma_{F}^{(0)}\right)}{\left|D_{F}\right|^{1 / 12}} & \text { if } k=0, \\ \frac{J_{1}\left(\sigma_{F}^{(1)}\right)}{\left|D_{F}\right|^{1 / 12}} & \text { if } k=1, \\ \frac{J_{2}\left(\sigma_{F}^{(2)}\right)}{\left|D_{F}\right|^{1 / 12}} & \text { if } k=2 .\end{cases}
$$

(The absolute value operation on the discriminant is unnecessary in the cases $k=0$ and $k=2$, but is included for consistency.)

This representation has several desirable properties:

(I) It is practical for computation (as we will soon explain).

(II) It expresses the quantity $\left|D_{F}\right|^{1 / 12} A_{F}$ in each of the cases $k=0,1,2$ as a function of a single parameter $\sigma_{F}$ which itself has the following desirable properties:

(a) $\sigma_{F}$ is invariant under $G L_{2}(\mathbb{R})$ (in the sense to be defined in $\S 3$ );

(b) $\sigma_{F} \in(0,1)$, so that $\left|D_{F}\right|^{1 / 12} A_{F}$ (when considered as a function of $\sigma_{F}$ ) is defined on a fixed bounded interval;

(c) $\sigma_{F}$ is invariant with respect to interchanging any single pair of complex conjugate roots $\alpha_{i} / \beta_{i}, \overline{\alpha_{i}} / \overline{\beta_{i}}$ (so we need not be concerned with an $a$ priori arrangement of the non-real roots).

(III) It is "straightforward" to derive using real linear fractional transformations and real quadratic transformations.

By contrast, the "standard" representation

$$
A_{F}=\frac{1}{2} \int_{0}^{2 \pi} \frac{d \theta}{|F(\cos \theta, \sin \theta)|^{2 / n}}
$$

\footnotetext{
${ }^{5}$ This is not true of $\rho_{F}$ which is only unique up to conjugation.
} 
(which follows directly from the polar form $r=|F(\cos \theta, \sin \theta)|^{-1 / n}$ of the curve $|F(x, y)|=1)$ is not practical for computation and does not describe $A_{F}$ in a meaningful way. It is quite conceivable that there are many other representations of $A_{F}$ which have the properties (I), (II), (III). However, for our present purposes, the representation given in Theorem 1 is certainly satisfactory.

An alternative representation which we will find particularly useful in our discussion below is the representation given by

$$
A_{F}= \begin{cases}\frac{J_{0}\left(\sigma_{F}^{(0)}\right)}{\left|D_{F}\right|^{1 / 12}} & \text { if } k=0, \\ \frac{J_{1}\left(\sigma_{F}^{(1)}\right)}{\left|D_{F}\right|^{1 / 12}} & \text { if } k=1, \\ \frac{\hat{J}_{2}\left(\hat{\sigma}_{F}^{(2)}\right)}{\left|D_{F}\right|^{1 / 12}} & \text { if } k=2,\end{cases}
$$

where $\hat{J}_{2}$ and $\hat{\sigma}_{F}^{(2)}$ are defined by

$$
\begin{aligned}
\hat{J}_{2}(x) & =2 x^{1 / 6}(1-x)^{1 / 6} I(x), \quad x \in(0,1), \\
\hat{\sigma}_{F}^{(2)} & =\frac{1}{\rho_{F}} \in(0,1)
\end{aligned}
$$

and where it is assumed that the $\alpha_{i} / \beta_{i}$ are arranged to satisfy (4) and the additional condition

$$
\left(\Im \frac{\alpha_{1}}{\beta_{1}}\right)\left(\Im \frac{\alpha_{3}}{\beta_{3}}\right)>0
$$

in the case $k=2$ (so that $\hat{\sigma}_{F}^{(2)} \in(0,1)$ ). Note that this representation differs from the representation of Theorem 1 only in the case $k=2$, but is "less desirable" since it fails to satisfy property (IIc) (in that case). We will not prove the correctness of the formula $A_{F}=\hat{J}_{2}\left(\hat{\sigma}_{F}^{(2)}\right) /\left|D_{F}\right|^{1 / 12}$ in the case $k=2$ since this formula is not used in the demonstration of any of our results. However, it can be derived without too much difficulty using complex integration techniques and complex fractional linear transformations.

We will now explain why the representation of Theorem 1 is practical for computation and how it can be implemented in practice. It is clear that this representation reduces the calculation of $A_{F}$ to the following basic computations:

(i) the algebraic computation of $D_{F}$ and $\sigma_{F}$;

(ii) the numeric computation of $z^{1 / 12}$ for $z>0$ and of $J_{k}(x)$ for $x \in(0,1)$.

We claim that each of these basic computations can be easily performed using standard mathematical software.

Consider first the algebraic computation of $D_{F}$ and $\sigma_{F}$. If a factorization $\prod_{i=1}^{4}\left(\alpha_{i} X-\beta_{i} Y\right)$ of $F(X, Y)$ is given, then we can compute $D_{F}$ and $\sigma_{F}$ directly from their definitions using elementary algebraic operations. On the other hand, if a factorization of $F(X, Y)$ is not given, we can always (effectively) obtain one from the coefficients of $F(X, Y)$ - using the formula for solving quartic polynomial equations if necessary to first find the roots of $F(x, 1)$ - and then calculate $D_{F}$ 
and $\sigma_{F}$ as before. In the latter case, i.e., the case where

$$
F(X, Y)=a_{0} X^{4}+a_{1} X^{3} Y+a_{2} X^{2} Y^{2}+a_{3} X Y^{3}+a_{4} Y^{4},
$$

it is usually better to compute the discriminant $D_{F}$ using the well known formula ${ }^{6}$

$$
\begin{aligned}
D_{F}=- & 80 a_{0} a_{1} a_{2}^{2} a_{3} a_{4}+144 a_{0}^{2} a_{2} a_{3}^{2} a_{4}-6 a_{0} a_{1}^{2} a_{3}^{2} a_{4}+18 a_{0} a_{1} a_{2} a_{3}^{3} \\
& -192 a_{0}^{2} a_{1} a_{3} a_{4}^{2}+144 a_{0} a_{1}^{2} a_{2} a_{4}^{2}-128 a_{0}^{2} a_{2}^{2} a_{4}^{2}-27 a_{0}^{2} a_{3}^{4} \\
& +16 a_{0} a_{2}^{4} a_{4}-4 a_{0} a_{2}^{3} a_{3}^{2}+18 a_{1}^{3} a_{2} a_{3} a_{4}-27 a_{2}^{4} a_{4}^{2} \\
& -4 a_{1}^{2} a_{2}^{3} a_{4}-4 a_{1}^{3} a_{3}^{3}+a_{1}^{2} a_{2}^{2} a_{3}^{2}+256 a_{0}^{3} a_{4}^{3}
\end{aligned}
$$

since this avoids the complexity of factoring $F(X, Y)$. In principle, it should also be possible to express $\sigma_{F}$ in terms of the coefficients of $F$. However, it is not clear that the resulting expression would involve any fewer computational steps than the routine of factoring $F(X, Y)$, computing $\rho_{F}$, and then computing $\sigma_{F}$ with (6). Indeed, it is not at all clear to us that $\rho_{F}$ (and hence $\sigma_{F}$ ) could even be effectively computed (using only elementary algebraic operations) if there were no formula for solving quartic polynomial equations. This suggests that there may be significant complications in the calculation of $A_{F}$ for forms of higher degree (see [2, Theorem 3]).

Now consider the numeric computation of $z^{1 / 12}$ and $J_{k}(x)$. Clearly the only potential difficulty here lies with the evaluation of the integrals $I(x)$. A glance at (7) reveals that each of the integrals $I(x)$ has singularities at the endpoints $z=0, z=1$ of the integration interval, but is otherwise well suited for numerical evaluation. We can easily remove the singularities of $I(x)$ by splitting the integral into two parts at the point $z=1 / 2$ and applying the respective substitutions $z=w^{2}$ and $z=1-w^{2}$. After doing this, we obtain the expression

$$
\begin{aligned}
I(x)=2 \int_{0}^{1 / \sqrt{2}} & \frac{d w}{\left(1-w^{2}\right)^{1 / 2}\left(1-x w^{2}\right)^{1 / 2}} \\
& +2 \int_{0}^{1 / \sqrt{2}} \frac{d w}{\left(1-w^{2}\right)^{1 / 2}\left(1-x+x w^{2}\right)^{1 / 2}}
\end{aligned}
$$

which can be readily evaluated using a standard numerical integration package. Combining these observations with the earlier ones concerning the calculation of $D_{F}$ and $\sigma_{F}$, it is then a straightforward matter to construct an algorithm for computing $A_{F}$ in practice.

It is worth mentioning that the function $I(x)$ is actually a scalar multiple of a complete elliptic integral of the first kind, and is the integral representation of a particular hypergeometric function. To be precise,

$$
I(x)=2 K(x)=\pi_{2} F_{1}\left(\frac{1}{2}, \frac{1}{2} ; 1 ; x\right)
$$

where $K(m)=\int_{0}^{\pi / 2}\left(1-m \sin ^{2} \theta\right)^{-1 / 2} d \theta$ is the complete elliptic integral of the first kind with parameter $m$ and ${ }_{2} F_{1}(a, b ; c ; x)$ is the ordinary hypergeometric function with coefficients $a, b$, and $c$ (see $[1$, p. 591]). Many commercially available software packages contain built-in elliptic integrals and hypergeometric functions. Hence (16) provides an alternative way to evaluate $I(x)$ in practice. It has been our experience, however, that these built-in functions sometimes fail to converge for

\footnotetext{
${ }^{6}$ This formula can be easily obtained using a computer algebra software package [6].
} 
values of $x$ close to 1 . In such cases, (15) can still be numerically integrated to obtain a value for $I(x)$.

While the representation of Theorem 1 is satisfactory for most purposes, it does have a couple of drawbacks. The most obvious drawback is the omission of the case $D_{F}=0$. However, this is not too serious since for such forms $F$, the area $A_{F}$ is infinite unless $F(X, Y)=Q(X, Y)^{2}$ for some definite quadratic form $Q$ in which case $A_{F}=2 \pi /\left|D_{Q}\right|^{1 / 2}$. The other drawback, which may be unavoidable, is the use of three separate formulas to describe $A_{F}$. It would be very satisfactory to have one ${ }^{7}$ formula for $A_{F}$ which covered all cases and exhibited properties similar to the properties (I), (II), (III) stated above. In light of equations (2) and (3) from $\S 1$, we suspect that this is not possible for the collection of quartic forms with real coefficients; however, it may be possible for the collection of quartic forms with complex coefficients.

Now let us consider the quantity $\left|D_{F}\right|^{1 / 12} A_{F}$ in greater detail. We already know from equation (1) of the Introduction that the maximum value of $\left|D_{F}\right|^{1 / 12} A_{F}$ over the class of quartic forms is $2^{7 / 6} B\left(\frac{1}{4}, \frac{1}{2}\right)$ and that $\left|D_{F}\right|^{1 / 12} A_{F}$ assumes all values between zero and its maximum. In light of equations (2) and (3), it is natural to ask how the maximum varies with the number of conjugate pairs of $F(x, 1)$. To facilitate this discussion, we introduce the following notation. Put

$$
M_{n, k}^{*}=\max \left|D_{F}\right|^{1 / n(n-1)} A_{F}
$$

where the maximum is taken over all binary forms $F$ of degree $n$ with real coefficients, non-zero discriminant, and with the property that the polynomial $F(x, 1)$ has exactly $k$ pairs of complex conjugate roots.

From the representations (9) and (11) of $A_{F}$, it is clear that in the case $n=4$ we have

$$
M_{4,2}^{*}<M_{4,1}^{*} \quad \text { and } \quad M_{4,2}^{*}<M_{4,0}^{*}
$$

since it is certainly true from the definitions of (8) and (12) of the functions $J_{0}, J_{1}$, $J_{2}, \hat{J}_{2}$ that

$$
J_{2}(x)<J_{1}(x) \text { and } \hat{J}_{2}(x)<J_{0}(x)
$$

for all $x \in(0,1)$. Together with equations (2) and (3) of the Introduction, this suggests that

$$
M_{4,2}^{*}<M_{4,1}^{*}<M_{4,0}^{*} .
$$

In fact, we will prove a much stronger result in $\S 4$.

Theorem 2. Put

$$
M_{n, k}=\max \left|D_{F}\right|^{1 / n(n-1)} A_{F}
$$

where the maximum is taken over all binary forms $F(X, Y)$ of degree $n$ with complex coefficients, non-zero discriminant, and with the property that the polynomial $F(x, 1)$ has exactly $k$ pairs of complex conjugate roots. Then for each $n \geq 2$,

$$
M_{n, 0}>M_{n, 1}>\cdots>M_{n,\left[\frac{n}{2}\right]} .
$$

Moreover, for each $k=0,1, \ldots,\left[\frac{n}{2}\right], M_{n, k}$ is attained by a form of degree $n$ with precisely $n-2 k$ real linear factors, the greatest number possible. In particular, $M_{n, k}$ is attained by a form with real coefficients.

\footnotetext{
${ }^{7}$ Such a formula need not contain $D_{F}$ explicitly.
} 
Since the $M_{n, k}$ are attained by forms with real coefficients, Theorem 2 remains true if we restrict our attention to this class of forms:

Corollary 1. Put

$$
M_{n, k}^{*}=\max \left|D_{F}\right|^{1 / n(n-1)} A_{F}
$$

where the maximum is taken over all binary forms $F(X, Y)$ of degree $n$ with real coefficients, non-zero discriminant, and with the property that $F(X, Y)$ has exactly $k$ definite quadratic factors in its factorization over $\mathbb{R}$. Then for all $n$ and $k$,

$$
M_{n, k}^{*}=M_{n, k} .
$$

Consequently, for each $n \geq 2$,

$$
M_{n, 0}^{*}>M_{n, 1}^{*}>\cdots>M_{n,\left[\frac{n}{2}\right]}^{*} .
$$

In particular,

$$
M_{4,2}^{*}<M_{4,1}^{*}<M_{4,0}^{*} .
$$

Theorem 2 also has the following consequence, which is essentially a restatement of Theorem 3 from [3]:

Corollary 2. The maximal value $M_{n}$ of the quantity $\left|D_{F}\right|^{1 / n(n-1)} A_{F}$ over the class of forms of degree $n$ with complex coefficients and non-zero discriminant is attained by a form $F$ with real coefficients for which the polynomial $F(x, 1)$ has $n$ distinct real roots. That is,

$$
M_{n}=M_{n, 0}^{*}
$$

for all $n$.

Consequently, Theorem 2 can be viewed as a generalization of this earlier result.

Theorem 2 raises several interesting questions regarding the nature of the sequences $\left\{M_{n, k}\right\}_{k=0}^{\left[\frac{n}{2}\right]}$ :

- What are the values of $M_{n, k}$ ?

- Are there canonical classes of forms $F_{n, k}$ for which

$$
M_{n, k}=\left|D_{F_{n, k}}\right|^{1 / n(n-1)} A_{F_{n, k}} ?
$$

- Is there a relationship among the sequences $\left\{M_{n, k}\right\}_{k=0}^{\left[\frac{n}{2}\right]}$ ?

- What is the "limiting behavior" of the $M_{n, k}$ as $n$ becomes large?

While we are unable to give complete answers to these questions at this time, we can give an indication of what the answers might be.

From equations (1), (2), and (3) of the Introduction, we clearly have ${ }^{8}$

$$
\begin{aligned}
& M_{2,0}=\infty \\
& M_{2,1}=2 \pi \approx 6.28318 \\
& M_{3,0}=3 B\left(\frac{1}{3}, \frac{1}{3}\right) \approx 15.89974, \\
& M_{3,1}=\sqrt{3} B\left(\frac{1}{3}, \frac{1}{3}\right) \approx 9.17972, \\
& M_{4,0}=2^{7 / 6} B\left(\frac{1}{4}, \frac{1}{2}\right) \approx 11.77264 ;
\end{aligned}
$$

\footnotetext{
${ }^{8}$ Numerical approximations are chopped, not rounded.
} 
moreover, we have a complete characterization of the forms $F_{n, k}$ for which these values of $M_{n, k}$ occur. We will show in $\S 5$ that

$$
\begin{aligned}
& M_{4,1} \approx 9.10746, \\
& M_{4,2} \approx 7.00758,
\end{aligned}
$$

using the formulas for $\left|D_{F}\right|^{1 / 12} A_{F}$ given in Theorem 1 and properties of the hypergeometric function. Unfortunately, we have not been able to determine canonical forms $F_{4,1}, F_{4,2}$ which give rise to these values. Consequently, the given values of $M_{4,1}$ and $M_{4,2}$ are numerical approximations only. ${ }^{9}$

When $n \geq 5$, we do not even have numerical approximations for the $M_{n, k}$. However, we do have some indication of what the values might be when $k=0$. From Theorem 2 and the fact that $\left\{M_{n}\right\}$ is decreasing, it is clear that the sequence $\left\{M_{n, 0}\right\}$ is also decreasing. On the basis of this fact and a correspondence between forms and equiangular polygons, we conjectured in [2] that

$$
M_{n, 0}=\left|D_{F_{n}^{*}}\right|^{1 / n(n-1)} A_{F_{n}^{*}}, \quad n \geq 5,
$$

where

$$
F_{n}^{*}(X, Y)=\prod_{j=1}^{n}\left(X \sin \left(\frac{j \pi}{n}\right)-Y \cos \left(\frac{j \pi}{n}\right)\right) .
$$

We also conjectured that $\lim _{n \rightarrow \infty} M_{n, 0}=2 \pi$. If these conjectures were true, then the sequence $\left\{M_{n, 0}\right\}$ would have a very natural characterization.

Unfortunately, there are no obvious candidates for $M_{n, k}$ when $k \neq 0$. Indeed, our analysis of the situation for quartic forms indicates that determining the values of $M_{n, k}$ in general could be quite difficult. It would appear from the known values of $M_{n, k}$ that $\left\{M_{n, 0}\right\}$ is the only sequence among those defined by fixing a value of $k$ which is decreasing.

It is not clear whether knowledge of the values of $M_{n, k}$ for $k \neq 0$ would have any immediate application. Nevertheless, it would be interesting to know the values of the sequence $\left\{M_{n,\left[\frac{n}{2}\right]}\right\}_{n=2}^{\infty}$, if only to compare them with the sequence $\left\{M_{n, 0}\right\}_{n=2}^{\infty}$. It is clear that $\lim _{n \rightarrow \infty} M_{n,\left[\frac{n}{2}\right]} \neq 0$; in fact, an examination of the forms $X^{n}+Y^{n}$ indicates that $\lim _{n \rightarrow \infty} M_{n,\left[\frac{n}{2}\right]} \geq 4$. One is tempted to conjecture that $\lim _{n \rightarrow \infty} M_{n,\left[\frac{n}{2}\right]}=2 \pi$, although at present there is not enough evidence to confidently predict that this is so. If one could show that $\lim _{n \rightarrow \infty} M_{n, 0}=2 \pi$ and $\lim _{n \rightarrow \infty} M_{n,\left[\frac{n}{2}\right]}=2 \pi$, then it would necessarily follow that $\lim _{n \rightarrow \infty} M_{n, k_{n}}=2 \pi$ for every sequence of integers $k_{n}$ satisfying $0 \leq k_{n} \leq\left[\frac{n}{2}\right]$.

\section{Formulas for calculating $A_{F}$}

In this section, we will derive the formulas given in the statement of Theorem 1 for calculating $A_{F}$ when $F$ is a quartic form. The practical implementation of these formulas was discussed in $\S 2$.

We begin by recalling some notation and some elementary facts from [3]. The facts stated below are actually true in the more general context of forms of degree $n$ with complex coefficients, and will be used in the proofs of both Theorem 1 and Theorem 2. (The proof of Theorem 2 will be given in the next section.)

\footnotetext{
${ }^{9}$ Nevertheless, the methods presented in $\S 5$ allow us (in principle) to determine the values of $M_{4,1}$ and $M_{4,2}$ to any desired accuracy.
} 
Let $G L_{2}(\mathbb{R})$ denote the group of $2 \times 2$ real invertible matrices. For any form $F(X, Y) \in \mathbb{C}[X, Y]$ and any $T=\left(\begin{array}{ll}a & b \\ c & d\end{array}\right) \in G L_{2}(\mathbb{R})$, let $F_{T}$ denote the form given by $F_{T}(X, Y)=F(a X+b Y, c X+d Y)$. We will say that two forms $F$ and $G$ are equivalent under $G L_{2}(\mathbb{R})$ if $G=F_{T}$ for some $T \in G L_{2}(\mathbb{R})$.

In [3], we showed that the quantity $\left|D_{F}\right|^{1 / n(n-1)} A_{F}$ is invariant under transformations in $G L_{2}(\mathbb{R})$, i.e. $\left|D_{F_{T}}\right|^{1 / n(n-1)} A_{F_{T}}=\left|D_{F}\right|^{1 / n(n-1)} A_{F}$ for all $T \in G L_{2}(\mathbb{R})$, and is invariant with respect to replacing $F$ by $\gamma F$ for any complex number $\gamma$. ${ }^{10}$ We also showed that $A_{F}$ has the integral representation

$$
A_{F}=\int_{-\infty}^{\infty} \frac{d u}{|F(u, 1)|^{2 / n}}=\int_{-\infty}^{\infty} \frac{d v}{|F(1, v)|^{2 / n}} .
$$

Moreover, we made the observation that a $G L_{2}(\mathbb{R})$ transformation can be specified (up to multiplication) by indicating its action on any three independent linear factors of a form $F(X, Y)$. Indeed, we noted that a $G L_{2}(\mathbb{R})$ transformation applied to any form $F(X, Y)$ induces a real fractional linear transformation of the roots of the polynomial $F(1, y)$, and that any real fractional linear transformation may be defined by the rule

$$
\frac{\left(w-w_{1}\right)\left(w_{3}-w_{2}\right)}{\left(w-w_{2}\right)\left(w_{3}-w_{1}\right)}=\frac{\left(z-z_{1}\right)\left(z_{3}-z_{2}\right)}{\left(z-z_{2}\right)\left(z_{3}-z_{1}\right)}
$$

where the $z$ 's and $w$ 's are real numbers such that $z_{1}, z_{2}, z_{3}$ are mapped to $w_{1}, w_{2}$, $w_{3}$ respectively. From these three facts, we concluded that a formula for calculating $A_{F}$ can be obtained by simply specifying three of the roots of $F_{T}(1, y)$, using (19) to calculate $A_{F_{T}}$, and then multiplying the result by $\left|D_{F_{T}} / D_{F}\right|^{1 / n(n-1)}$.

Now suppose that $F(X, Y)=\prod_{i=1}^{4}\left(\alpha_{i} X-\beta_{i} X\right)$ is a binary quartic form with real coefficients and non-zero discriminant, and let $k$ be the number of pairs of complex conjugate roots of the polynomial $F(x, 1)$. ( $k$ is also the number of pairs of complex conjugate roots of $F(1, y)$.) Suppose further that the fractions $\alpha_{i} / \beta_{i}$ are arranged according to (4) in $\S 2$, and let the quantities $\rho_{F}$ and $\sigma_{F}^{(k)}$ be defined by (5) and (6) respectively. Let the functions $I$ and $J_{k}$ be defined by (7) and (8) respectively. We will derive the representations for $A_{F}$ stated in Theorem 1 by separately considering the cases $k=0, k=1$, and $k=2$.

In each of these three cases, our strategy will be the same. First, we will use a suitable transformation from $G L_{2}(\mathbb{R})$ to reduce the calculation of $\left|D_{F}\right|^{1 / 12} A_{F}$ in general to its calculation over a class of "canonical" forms $F^{(t)}$ which depends on a single real parameter $t$. Then, we will use the integral representation given in (19) above to obtain an explicit formula for $\left|D_{F^{(t)}}\right|^{1 / 12} A_{F^{(t)}}$. Finally, we will use a suitable quadratic or linear fractional transformation to transform the resulting integrals into integrals of hypergeometric type. In each case, the initial $G L_{2}(\mathbb{R})$ transformation will be chosen to ensure that the subsequent calculations lead to the desired representation of $A_{F}$.

Case 1: $k=0$. In this case, the polynomial $F(x, 1)$ has four real roots (counting any root at infinity).

\footnotetext{
${ }^{10}$ Note that $\left|D_{F}\right|^{1 / n(n-1)} A_{F}$ is not invariant with respect to $G L_{2}(\mathbb{C})$. See, for example, equation (2) of $\S 1$.
} 
There is a real fractional linear transformation of the roots such that

$$
\frac{\alpha_{1}}{\beta_{1}} \mapsto-\infty, \frac{\alpha_{2}}{\beta_{2}} \mapsto 0, \quad \frac{\alpha_{3}}{\beta_{3}} \mapsto t, \quad \frac{\alpha_{4}}{\beta_{4}} \mapsto 1, \quad t \in(0,1)
$$

and so every form $F$ in this class is equivalent under $G L_{2}(\mathbb{R})$ to a form of the type

$$
F^{(t)}(X, Y)= \pm X Y(Y-X)(Y-t X), \quad t \in(0,1) .
$$

(Note that the statement $t \in(0,1)$ is actually a consequence of our assignment of $\alpha_{1} / \beta_{1}, \alpha_{2} / \beta_{2}, \alpha_{4} / \beta_{4}$ since real fractional linear transformations preserve or reverse the cyclic order of the real numbers.) Under such a transformation

$$
\rho_{F}=t \text {. }
$$

Using the representation (19), we find that

$$
\begin{aligned}
A_{F^{(t)}}= & \int_{-\infty}^{0} \frac{d v}{(-v)^{1 / 2}(1-v)^{1 / 2}(1-t v)^{1 / 2}}+\int_{0}^{1} \frac{d v}{v^{1 / 2}(1-v)^{1 / 2}(1-t v)^{1 / 2}} \\
& \quad+\int_{1}^{1 / t} \frac{d v}{v^{1 / 2}(v-1)^{1 / 2}(1-t v)^{1 / 2}}+\int_{1 / t}^{\infty} \frac{d v}{v^{1 / 2}(v-1)^{1 / 2}(t v-1)^{1 / 2}} \\
= & 2 \int_{0}^{1} \frac{d w}{w^{1 / 2}(1-w)^{1 / 2}(1-t w)^{1 / 2}}+2 \int_{0}^{1} \frac{d w}{w^{1 / 2}(1-w)^{1 / 2}(1-(1-t) w)^{1 / 2}} .
\end{aligned}
$$

(The latter integrals follow from the former by applying the respective substitutions $v=-w /(1-w), v=w, v=(1-(1-t) w) / t, v=1 /(t w)$.) Hence

$$
\left|D_{F^{(t)}}\right|^{1 / 12} A_{F^{(t)}}=2 t^{1 / 6}(1-t)^{1 / 6}(I(t)+I(1-t)) .
$$

Now from (20) and the definition of $\sigma_{F}^{(0)}$, we have $\sigma_{F}^{(0)}=t$. Consequently, if $F$ is any form in the class $k=0$, then

$$
\left|D_{F}\right|^{1 / 12} A_{F}=J_{0}\left(\sigma_{F}^{(0)}\right)
$$

where

$$
J_{0}(x)=2 x^{1 / 6}(1-x)^{1 / 6}(I(x)+I(1-x)) .
$$

(This formula is actually a special case of Theorem 3 from [2]. The derivation is presented here for completeness.)

Case 2: $k=1$. In this case, the polynomial $F(x, 1)$ has two real roots and one pair of complex conjugate roots.

There is a real fractional linear transformation of the roots such that

$$
\frac{\alpha_{1}}{\beta_{1}} \mapsto-1, \quad \frac{\alpha_{2}}{\beta_{2}} \mapsto 1, \quad \frac{\alpha_{3}}{\beta_{3}} \mapsto i t, \quad \frac{\alpha_{4}}{\beta_{4}} \mapsto-i t, \quad t>0,
$$

and so every form $F$ in this class is equivalent under $G L_{2}(\mathbb{R})$ to a form of the type

$$
F^{(t)}(X, Y)=\left(Y^{2}-X^{2}\right)\left(Y^{2}+t^{2} X^{2}\right), \quad t>0 .
$$

Under such a transformation,

$$
\rho_{F}=\left(\frac{1-i t}{1+i t}\right)^{2} .
$$


By the symmetry of $F^{(t)}$, and using the representation (19), we have

$$
A_{F^{(t)}}=2 \int_{0}^{1} \frac{d v}{\left(1-v^{2}\right)^{1 / 2}\left(v^{2}+t^{2}\right)^{1 / 2}}+2 \int_{1}^{\infty} \frac{d v}{\left(v^{2}-1\right)^{1 / 2}\left(v^{2}+t^{2}\right)^{1 / 2}} .
$$

Further

$$
\int_{0}^{1} \frac{d v}{\left(1-v^{2}\right)^{1 / 2}\left(v^{2}+t^{2}\right)^{1 / 2}}=\frac{1}{2\left(1+t^{2}\right)^{1 / 2}} \int_{0}^{1} \frac{d w}{w^{1 / 2}(1-w)^{1 / 2}\left(1-\frac{1}{1+t^{2}} w\right)^{1 / 2}}
$$

(using the substitution $v=\sqrt{1-w}$ ) and

$$
\int_{1}^{\infty} \frac{d v}{\left(v^{2}-1\right)^{1 / 2}\left(v^{2}+t^{2}\right)^{1 / 2}}=\frac{1}{2\left(1+t^{2}\right)^{1 / 2}} \int_{0}^{1} \frac{d w}{w^{1 / 2}(1-w)^{1 / 2}\left(1-\frac{t^{2}}{1+t^{2}} w\right)^{1 / 2}}
$$

(using the substitution $v=1 / \sqrt{1-w}$ ). Hence

$$
\begin{aligned}
\left|D_{F^{(t)}}\right|^{1 / 12} A_{F^{(t)}} & =\frac{2^{1 / 3} t^{1 / 6}}{\left(1+t^{2}\right)^{1 / 6}}\left\{I\left(\frac{1}{1+t^{2}}\right)+I\left(\frac{t^{2}}{1+t^{2}}\right)\right\} \\
& =2^{1 / 3} s^{1 / 12}(1-s)^{1 / 12}(I(s)+I(1-s))
\end{aligned}
$$

where $s=1 /\left(1+t^{2}\right)$.

We claim that $s=\frac{1}{2}\left(1 \pm \sqrt{\left(1+\Re \rho_{F}\right) / 2}\right)$. From (21) we have $\rho_{F}=e^{i \zeta}$ for some $\zeta \in(-\pi, \pi)$ and so $(1-i t) /(1+i t)= \pm e^{i \zeta / 2}$. Suppose that $(1-i t) /(1+i t)=e^{i \zeta / 2}$. Then $t=-i\left(1-e^{i \zeta / 2}\right) /\left(1+e^{i \zeta / 2}\right)=-\left(\sin \frac{\zeta}{2}\right) /\left(1+\cos \frac{\zeta}{2}\right)$ and so $s=1 /\left(1+t^{2}\right)=$ $\left(1+\cos \frac{\zeta}{2}\right) / 2$. On the other hand, if $(1-i t) /(1+i t)=-e^{i \zeta / 2}$, then $s=\left(1-\cos \frac{\zeta}{2}\right) / 2$. Now $\cos \frac{\zeta}{2}=\sqrt{(1+\cos \zeta) / 2}$. Consequently, $s=\frac{1}{2}\left(1 \pm \sqrt{\left(1+\Re \rho_{F}\right) / 2}\right)$ as claimed.

Now $s=\frac{1}{2}\left(1 \pm \sqrt{\left(1+\Re \rho_{F}\right) / 2}\right)$ if and only if $1-s=\frac{1}{2}\left(1 \mp \sqrt{\left(1+\Re \rho_{F}\right) / 2}\right)$. Hence $\sigma_{F}^{(1)}$, which is defined to be $\frac{1}{2}\left(1+\sqrt{\left(1+\Re \rho_{F}\right) / 2}\right)$, is either $s$ or $1-s$. Since $\left|D_{F^{(t)}}\right|^{1 / 12} A_{F^{(t)}}$ is invariant with respect to interchanging $s$ and $1-s$, it follows that

$$
\left|D_{F^{(t)}}\right|^{1 / 12} A_{F^{(t)}}=2^{1 / 3}\left(\sigma_{F}^{(1)}\right)^{1 / 12}\left(1-\sigma_{F}^{(1)}\right)^{1 / 12}\left(I\left(\sigma_{F}^{(1)}\right)+I\left(1-\sigma_{F}^{(1)}\right)\right) .
$$

Consequently, if $F$ is any form in the class $k=1$, then

$$
\left|D_{F}\right|^{1 / 12} A_{F}=J_{1}\left(\sigma_{F}^{(1)}\right)
$$

where

$$
J_{1}(x)=2^{1 / 3} x^{1 / 12}(1-x)^{1 / 12}(I(x)+I(1-x)) .
$$

Case 3: $k=2$. In this case, the polynomial $F(x, 1)$ has two pairs of complex conjugate roots.

There is a real fractional linear transformation of the roots such that

$$
\frac{\alpha_{1}}{\beta_{1}} \mapsto-i, \frac{\alpha_{2}}{\beta_{2}} \mapsto i, \frac{\alpha_{3}}{\beta_{3}} \mapsto-i t, \frac{\alpha_{4}}{\beta_{4}} \mapsto i t, \quad t \in(0,1),
$$

and so every form $F$ in this class is equivalent under $G L_{2}(\mathbb{R})$ to a form of the type

$$
F^{(t)}(X, Y)= \pm\left(Y^{2}+X^{2}\right)\left(Y^{2}+t^{2} X^{2}\right) \quad t \in(0,1) .
$$


Under such a transformation,

$$
\rho_{F}=\left(\frac{1+t}{1-t}\right)^{2}>1
$$

By the symmetry of $F^{(t)}$, and using the representation (19), we have

$$
\begin{aligned}
A_{F^{(t)}} & =2 \int_{0}^{\infty} \frac{d v}{\left(v^{2}+1\right)^{1 / 2}\left(v^{2}+t^{2}\right)^{1 / 2}} \\
& =\int_{0}^{1} \frac{d w}{w^{1 / 2}(1-w)^{1 / 2}\left(1-\left(1-t^{2}\right) w\right)^{1 / 2}} .
\end{aligned}
$$

(The latter integral follows from the former using the substitution $v=\sqrt{1 / w-1}$.) Hence

$$
\left|D_{F^{(t)}}\right|^{1 / 12} A_{F^{(t)}}=2^{1 / 3} t^{1 / 6}\left(1-t^{2}\right)^{1 / 3} I\left(1-t^{2}\right) .
$$

From (22) and the definition of $\sigma_{F}^{(2)}$, it is straightforward to show that

$$
t^{2}=\left(\frac{\sqrt{\rho_{F}}-1}{\sqrt{\rho_{F}}+1}\right)^{2}=\sigma_{F}^{(2)} .
$$

Consequently, if $F$ is any form in the class $k=2$, then

$$
\left|D_{F}\right|^{1 / 12} A_{F}=J_{2}\left(\sigma_{F}^{(2)}\right)
$$

where

$$
J_{2}(x)=2^{1 / 3} x^{1 / 12}(1-x)^{1 / 3} I(1-x) .
$$

This completes the proof of Theorem 1 .

\section{The nature of the Sequences $\left\{M_{n, k}\right\}$}

Put

$$
M_{n, k}=\max \left|D_{F}\right|^{1 / n(n-1)} A_{F}
$$

where the maximum is taken over all binary forms $F$ of degree $n$ with complex coefficients, non-zero discriminant, and with the property that the polynomial $F(x, 1)$ has exactly $k$ pairs of complex conjugate roots. In this section, we will show that for each $n \geq 2$,

$$
M_{n, 0}>M_{n, 1}>\cdots>M_{n,\left[\frac{n}{2}\right]} .
$$

We will also show that for each $k=0,1, \ldots,\left[\frac{n}{2}\right], M_{n, k}$ is attained by a form of degree $n$ with precisely $n-2 k$ real linear factors, the greatest number possible. Theorem 2 and its corollaries will then follow.

We remarked in the previous section that the quantity $\left|D_{F}\right|^{1 / n(n-1)} A_{F}$ is invariant with respect to $G L_{2}(\mathbb{R})$ and with respect to replacing $F$ by $\gamma F$ for any complex number $\gamma$. Hence, when analyzing $\left|D_{F}\right|^{1 / n(n-1)} A_{F}$, we need only consider the quantity

$$
Q\left(\gamma_{1}, \ldots, \gamma_{n}\right)=\prod_{i<j}\left|\gamma_{j}-\gamma_{i}\right|^{2 / n(n-1)} \int_{-\infty}^{\infty} \frac{d v}{\left|\left(v-\gamma_{1}\right) \cdots\left(v-\gamma_{n}\right)\right|^{2 / n}}
$$


over all $n$-tuples $\left(\gamma_{1}, \ldots, \gamma_{n}\right)$ of distinct complex numbers. We will adopt the convention that if one of these $\gamma$ 's is infinite, say $\gamma_{n}$, then

$$
Q\left(\gamma_{1}, \ldots, \gamma_{n}\right)=\prod_{1 \leq i<j \leq n-1}\left|\gamma_{j}-\gamma_{i}\right|^{2 / n(n-1)} \int_{-\infty}^{\infty} \frac{d v}{\left|\left(v-\gamma_{1}\right) \cdots\left(v-\gamma_{n-1}\right)\right|^{2 / n}}
$$

To simplify the discussion which follows, we introduce the following notation. Let $\mathcal{C}_{n, k}$ be the collection of $n$-tuples $\left(\gamma_{1}, \ldots, \gamma_{n}\right)$ of complex numbers with the following properties:

(i) the numbers $\gamma_{1}, \ldots, \gamma_{n}$ are all distinct;

(ii) there are exactly $k$ complex conjugate pairs among the numbers $\gamma_{1}, \ldots, \gamma_{n}$.

Further, let $\mathcal{C}_{n, k}^{*}$ be the subset of $\mathcal{C}_{n, k}$ whose elements $\left(\gamma_{1}, \ldots, \gamma_{n}\right)$ satisfy the following additional property:

(iii) any component $\eta$ of $\left(\gamma_{1}, \ldots, \gamma_{n}\right)$ whose complex conjugate is not one of the other components of $\left(\gamma_{1}, \ldots, \gamma_{n}\right)$ must be a real number.

Then

$$
M_{n, k}=\max _{\left(\gamma_{1}, \ldots, \gamma_{n}\right) \in \mathcal{C}_{n, k}} Q\left(\gamma_{1}, \ldots, \gamma_{n}\right)
$$

and

$$
M_{n, k}^{*}=\max _{\left(\gamma_{1}, \ldots, \gamma_{n}\right) \in \mathcal{C}_{n, k}^{*}} Q\left(\gamma_{1}, \ldots, \gamma_{n}\right) .
$$

(Recall that $M_{n, k}^{*}$ was defined in $\S 2$ to be the maximum value of $\left|D_{F}\right|^{1 / n(n-1)} A_{F}$ over all binary forms $F$ of degree $n$ with real coefficients, non-zero discriminant, and for which the polynomial $F(x, 1)$ has exactly $k$ pairs of complex conjugate roots.) We will show that

$$
\left(\gamma_{1}, \ldots, \gamma_{n}\right) \in \mathcal{C}_{n, k+1} \Longrightarrow Q\left(\gamma_{1}, \ldots, \gamma_{n}\right)<M_{n, k}
$$

and that

$$
\left(\gamma_{1}, \ldots, \gamma_{n}\right) \in \mathcal{C}_{n, k} \backslash \mathcal{C}_{n, k}^{*} \Longrightarrow Q\left(\gamma_{1}, \ldots, \gamma_{n}\right)<M_{n, k}^{*}
$$

and Theorem 2 will follow.

Before discussing the details of the proof, let us recall the following terminology from the theory of complex functions. A continuous real-valued function $u$ of a single complex variable $z=x+i y$ is harmonic if it has continuous partial derivatives of the second order and satisfies Laplace's equation $\partial^{2} u / \partial x^{2}+\partial^{2} u / \partial y^{2}=0$. A continuous real-valued function $v$ of a single complex variable is said to be subharmonic if, in any region of the complex plane, $v$ is less than or equal to the harmonic function $u$ which coincides with $v$ on the boundary of the region. A subharmonic function need not be continuous; however, this assumption allows one to simplify the definition to some extent.

An important property of subharmonic functions is that they satisfy a maximum principle. The maximum principle for subharmonic functions states that a non-constant subharmonic function has no maximum in its region of definition. Consequently, the maximum of a subharmonic function on a closed bounded set is attained on the boundary of the set.

The generalizations of these concepts to functions of several complex variables are respectively the notions of pluriharmonicity and plurisubharmonicity. A continuous real-valued function of several complex variables is said to be plurisubharmonic if its restriction to any complex line is subharmonic on that line. The function is 
pluriharmonic if its restriction to any complex line is harmonic on that line. (A complex line in $\mathbb{C}^{n}$ is a set of the form $\{a+b \zeta: \zeta \in \mathbb{C}\}$ where $a, b \in \mathbb{C}^{n}$.) There is also a maximum principle for plurisubharmonic functions analogous to the one for subharmonic functions. (For details, see [7].)

Now consider the quantity $Q\left(\gamma_{1}, \ldots, \gamma_{n}\right)$. In [3], we showed that $Q\left(\gamma_{1}, \ldots, \gamma_{n}\right)$ is plurisubharmonic on the region

$$
\mathcal{R}=\mathbb{C}^{n} \backslash \bigcup_{i=1}^{n}\left\{\left(\gamma_{1}, \ldots, \gamma_{n}\right) \in \mathbb{C}^{n}: \gamma_{i} \in \mathbb{R}\right\}
$$

We will prove (23) and (24) by successively applying the maximum principle for plurisubharmonic functions.

First suppose that $\left(\gamma_{1}, \ldots, \gamma_{n}\right) \in \mathcal{C}_{n, k+1}$. To distinguish between the $\gamma_{i}$ 's which belong to a conjugate pair and those which do not, we will write $\alpha_{1}, \overline{\alpha_{1}}, \ldots, \alpha_{k+1}$, $\overline{\alpha_{k+1}}$ for the conjugate pairs and $\beta_{1}, \ldots, \beta_{n-2(k+1)}$ for the rest; if we wish to emphasize that a number (such as a $\beta_{j}$ ) is real, we will write $r_{j}$. By re-arranging the $\gamma_{i}$ 's, if necessary, we may assume that

$$
\left(\gamma_{1}, \ldots, \gamma_{n}\right)=\left(\alpha_{1}, \overline{\alpha_{1}}, \ldots, \alpha_{k}, \overline{\alpha_{k}}, \alpha_{k+1}, \overline{\alpha_{k+1}}, \beta_{1}, \ldots, \beta_{n-2(k+1)}\right)
$$

(since $Q\left(\gamma_{1}, \ldots, \gamma_{n}\right)$ is invariant under all permutations of $\left.\gamma_{1}, \ldots, \gamma_{n}\right)$.

Suppose that we fix $\alpha_{1}, \ldots, \alpha_{k}, \beta_{1}, \ldots, \beta_{n-2(k+1)}$ and consider the quantity $Q\left(\alpha_{1}, \overline{\alpha_{1}}, \ldots, \alpha_{k}, \overline{\alpha_{k}}, \gamma, \delta, \beta_{1}, \ldots, \beta_{n-2(k+1)}\right)$ over all $\gamma, \delta \in \mathbb{C}$ distinct from the $\alpha_{i}, \beta_{j}$. (Note that $\gamma, \delta$ need not be a conjugate pair here.) Then by the maximum principle for plurisubharmonic functions,

$$
\begin{aligned}
& Q\left(\alpha_{1}, \overline{\alpha_{1}}, \ldots, \alpha_{k}, \overline{\alpha_{k}}, \gamma, \delta, \beta_{1}, \ldots, \beta_{n-2(k+1)}\right) \\
& \quad \leq Q\left(\alpha_{1}, \overline{\alpha_{1}}, \ldots, \alpha_{k}, \overline{\alpha_{k}}, r_{1}, r_{2}, \beta_{1}, \ldots, \beta_{n-2(k+1)}\right)
\end{aligned}
$$

for some real numbers $r_{1}, r_{2}$; moreover, the inequality is strict if at least one of $\gamma$, $\delta$ is non-real. Consequently,

$$
\begin{aligned}
& Q\left(\alpha_{1}, \overline{\alpha_{1}}, \ldots, \alpha_{k}, \overline{\alpha_{k}}, \alpha_{k+1}, \overline{\alpha_{k+1}}, \beta_{1}, \ldots, \beta_{n-2(k+1)}\right) \\
& \quad<Q\left(\alpha_{1}, \overline{\alpha_{1}}, \ldots, \alpha_{k}, \overline{\alpha_{k}}, r_{1}, r_{2}, \beta_{1}, \ldots, \beta_{n-2(k+1)}\right) \\
& \quad \leq M_{n, k} .
\end{aligned}
$$

Hence, if $\left(\gamma_{1}, \ldots, \gamma_{n}\right) \in \mathcal{C}_{n, k+1}$, then $Q\left(\gamma_{1}, \ldots, \gamma_{n}\right)<M_{n, k}$ as claimed in (23).

Now suppose that $\left(\gamma_{1}, \ldots, \gamma_{n}\right) \in \mathcal{C}_{n, k} \backslash \mathcal{C}_{n, k}^{*}$. As in the previous paragraph, we can assume that $\left(\gamma_{1}, \ldots, \gamma_{n}\right)=\left(\alpha_{1}, \overline{\alpha_{1}}, \ldots, \alpha_{k}, \overline{\alpha_{k}}, \beta_{1}, \ldots, \beta_{n-2 k}\right)$ where the $\alpha_{i}$ are all non-real and the $\beta_{j}$ do not have conjugates among the other components. Suppose that we fix all the $\alpha_{i}, \beta_{j}$ except for $\beta_{1}$. Then by the maximum principle for subharmonic functions,

$$
Q\left(\alpha_{1}, \overline{\alpha_{1}}, \ldots, \alpha_{k}, \overline{\alpha_{k}}, \beta_{1}, \ldots, \beta_{n-2 k}\right)<Q\left(\alpha_{1}, \overline{\alpha_{1}}, \ldots, \alpha_{k}, \overline{\alpha_{k}}, r_{1}, \beta_{2}, \ldots, \beta_{n-2 k}\right)
$$

for some real number $r_{1}$. Continuing in this way, we find that

$$
\begin{aligned}
Q\left(\alpha_{1}, \overline{\alpha_{1}}, \ldots, \alpha_{k}, \overline{\alpha_{k}}, \beta_{1}, \ldots, \beta_{n-2 k}\right) & <Q\left(\alpha_{1}, \overline{\alpha_{1}}, \ldots, \alpha_{k}, \overline{\alpha_{k}}, r_{1}, \ldots, r_{n-2 k}\right) \\
& \leq M_{n, k}^{*}
\end{aligned}
$$

(for some real numbers $\left.r_{1}, \ldots, r_{n-2 k}\right)$. Consequently, if $\left(\gamma_{1}, \ldots, \gamma_{n}\right) \in \mathcal{C}_{n, k} \backslash \mathcal{C}_{n, k}^{*}$, then $Q\left(\gamma_{1}, \ldots, \gamma_{n}\right)<M_{n, k}^{*}$ as claimed in (24).

Now from (23), it follows, by induction on $k$, that

$$
M_{n, 0}>M_{n, 1}>\cdots>M_{n,\left[\frac{n}{2}\right]} ;
$$


and from (24), it follows that each $M_{n, k}$ is attained by a form $F$ of degree $n$ with real coefficients for which the polynomial $F(x, 1)$ has precisely $n-2 k$ real roots (counting any root at infinity).

This completes the proof of Theorem 2. Corollary 1 and Corollary 2 follow immediately.

\section{Numerical VAlues fOr $\left\{M_{4, k}\right\}$}

We conclude this paper by giving numerical values for $\left\{M_{4, k}\right\}_{k=0}^{2}$. In particular, we will show that

$$
M_{4,1} \approx 9.10746
$$

and

$$
M_{4,2} \approx 7.00758
$$

(The value of $M_{4,0}$ was previously determined in [2] to be $2^{7 / 6} B\left(\frac{1}{4}, \frac{1}{2}\right) \approx 11.77264$.) Unfortunately, we have not been able to determine canonical quartic forms $F_{4,1}$, $F_{4,2}$ which give rise to these values. Hence, the values of $M_{4,1}$ and $M_{4,2}$ given are numerical approximations only. Nevertheless, we will see that $M_{4,1}$ and $M_{4,2}$ can be determined (in principle) to any desired accuracy.

In view of Theorem 1 , we have

$$
M_{4, k}=\max _{x \in(0,1)} J_{k}(x)
$$

where

$$
\begin{aligned}
& J_{0}(x)=2 x^{1 / 6}(1-x)^{1 / 6}(I(x)+I(1-x)), \\
& J_{1}(x)=2^{1 / 3} x^{1 / 12}(1-x)^{1 / 12}(I(x)+I(1-x)), \\
& J_{2}(x)=2^{1 / 3} x^{1 / 12}(1-x)^{1 / 3} I(1-x),
\end{aligned}
$$

and where

$$
I(x)=\int_{0}^{1} \frac{d z}{z^{1 / 2}(1-z)^{1 / 2}(1-x z)^{1 / 2}} .
$$

Hence, to determine the values of $M_{4, k}$, we need only analyze the functions $J_{k}(x)$.

Now the simplest way to get a sense of the behavior of the $J_{k}$ is to plot their graphs. Initial plots of $J_{1}$ and $J_{2}$ suggest that the maxima occur near one (or both) of the endpoints of the interval $(0,1)$; magnifications of $J_{1}$ and $J_{2}$ near $x=0$ confirm that this is indeed the case. (See Figures 2 and 3.)

From the graphs of $J_{1}$ and $J_{2}$, we surmise that $J_{1}$ and $J_{2}$ have the following properties:

(i) $J_{k}(x) \rightarrow 0$ as $x \rightarrow 0$ or 1 ;

(ii) $J_{1}$ has exactly three critical points: one local minimum at $x=\frac{1}{2}$ and two global maxima (one near each endpoint); moreover, $J_{1}$ is symmetric about $x=\frac{1}{2}$;

(iii) $J_{2}$ has exactly one critical point - a global maximum near the endpoint $x=0$. 


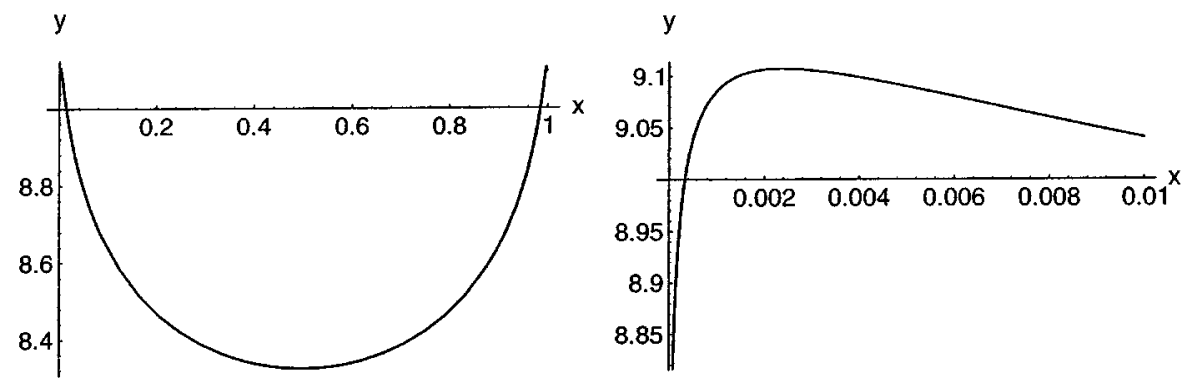

FiguRE 2. Graphs of $J_{1}$

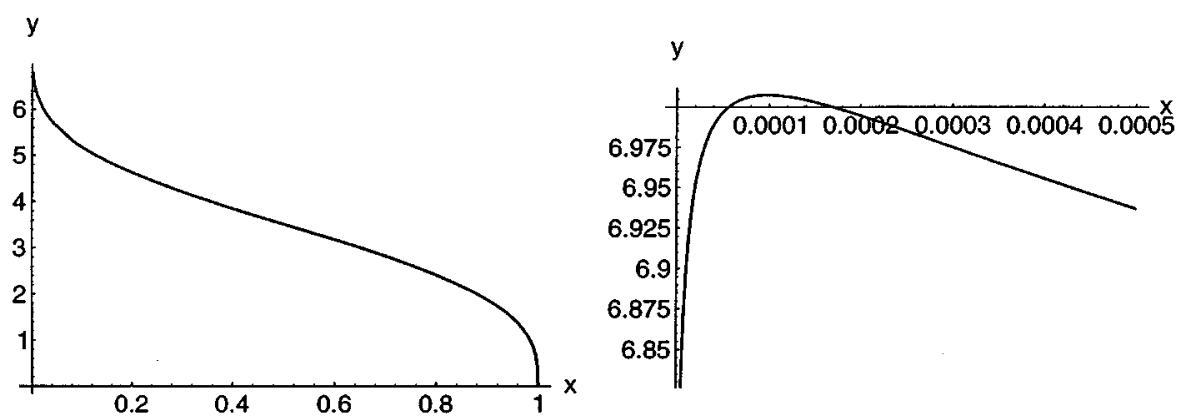

FiguRE 3. Graphs of $J_{2}$

TABLE 1. Numerical values of $J_{1}$ near its maximum

\begin{tabular}{|cc|}
\hline$x$ & $J_{1}(x)$ \\
\hline .002365 & 9.107465779 \\
.002366 & 9.107465839 \\
.002367 & 9.107465888 \\
.002368 & 9.107465926 \\
.002369 & 9.107465953 \\
.002370 & 9.107465970 \\
.002371 & 9.107465975 \\
.002372 & 9.107465970 \\
.002373 & 9.107465953 \\
.002374 & 9.107465926 \\
.002375 & 9.107465888 \\
\hline
\end{tabular}

Assuming these properties are true, we can determine the values of $M_{4,1}$ and $M_{4,2}$ to any desired accuracy by computing values of $J_{1}(x)$ and $J_{2}(x)$ on either side of their respective maximum points. ${ }^{11}$ The results of such computations are given in Table 1 and Table 2.

\footnotetext{
${ }^{11}$ Of course, when numerically evaluating $I(x)$, we will use the form of $I(x)$ given by $(15)$ or (16) in $\S 2$.
} 
TABLE 2. Numerical values of $J_{2}$ near its maximum

\begin{tabular}{|cc|}
\hline$x$ & $J_{2}(x)$ \\
\hline .0000975 & 7.007579498 \\
.0000976 & 7.007579794 \\
.0000977 & 7.007580038 \\
.0000978 & 7.007580231 \\
.0000979 & 7.007580372 \\
.0000980 & 7.007580463 \\
.0000981 & 7.007580503 \\
.0000982 & 7.007580493 \\
.0000983 & 7.007580432 \\
.0000984 & 7.007580320 \\
.0000985 & 7.007580159 \\
\hline
\end{tabular}

From these tables, it appears that

$$
\begin{aligned}
& M_{4,1} \approx 9.10746, \\
& M_{4,2} \approx 7.00758,
\end{aligned}
$$

as earlier claimed.

Before we can say with certainty that these values are correct to the given number of decimal places, we must show that properties (i), (ii), (iii) of $J_{1}$ and $J_{2}$ stated above actually hold. In particular, we must show that the graphs in Figures 2 and 3 are accurate representations of the functions $J_{1}, J_{2}$ near $x=0$, i.e., we must show that the software used to generate the graphs has not inadvertently concealed some singularity. The only way to do this is to give an analytic proof of properties (i), (ii), and (iii).

It is convenient to prove properties (i), (ii), (iii) for a slightly more general class of functions.

Lemma 1. Consider the family of functions $S_{r}$, for $r>0$, defined by

$$
S_{r}(x)=x^{r}(1-x)^{r}(I(x)+I(1-x)), \quad x \in(0,1) .
$$

The behavior of $S_{r}(x)$ depends on whether $r \geq \frac{1}{8}$ or $r<\frac{1}{8}$. If $r \geq \frac{1}{8}$, then $S_{r}(x)$ is increasing for $x \in\left(0, \frac{1}{2}\right)$ and decreasing for $x \in\left(\frac{1}{2}, 1\right)$; hence $S_{r}(x)$ has exactly one critical point - a global maximum point at $x=\frac{1}{2}$. On the other hand, if $r<\frac{1}{8}$, then $S_{r}(x)$ has exactly three critical points and $x=\frac{1}{2}$ is a local minimum point. In either case, $S_{r}(x)$ approaches zero as $x$ approaches the endpoints of the interval $(0,1)$.

Lemma 2. Consider the family of functions $T_{r, s}$, for $r>0$ and $s>0$, defined by

$$
T_{r, s}(x)=x^{r}(1-x)^{s} I(x), \quad x \in(0,1) .
$$

If $3 r+4 s>1$, then $T_{r, s}(x)$ has exactly one critical point which is a global maximum point. Moreover, $T_{r, s}(x)$ approaches zero as $x$ approaches either endpoint of the interval $(0,1)$.

Remark. It may be possible to weaken the condition $3 r+4 s>1$ in Lemma 2 . 

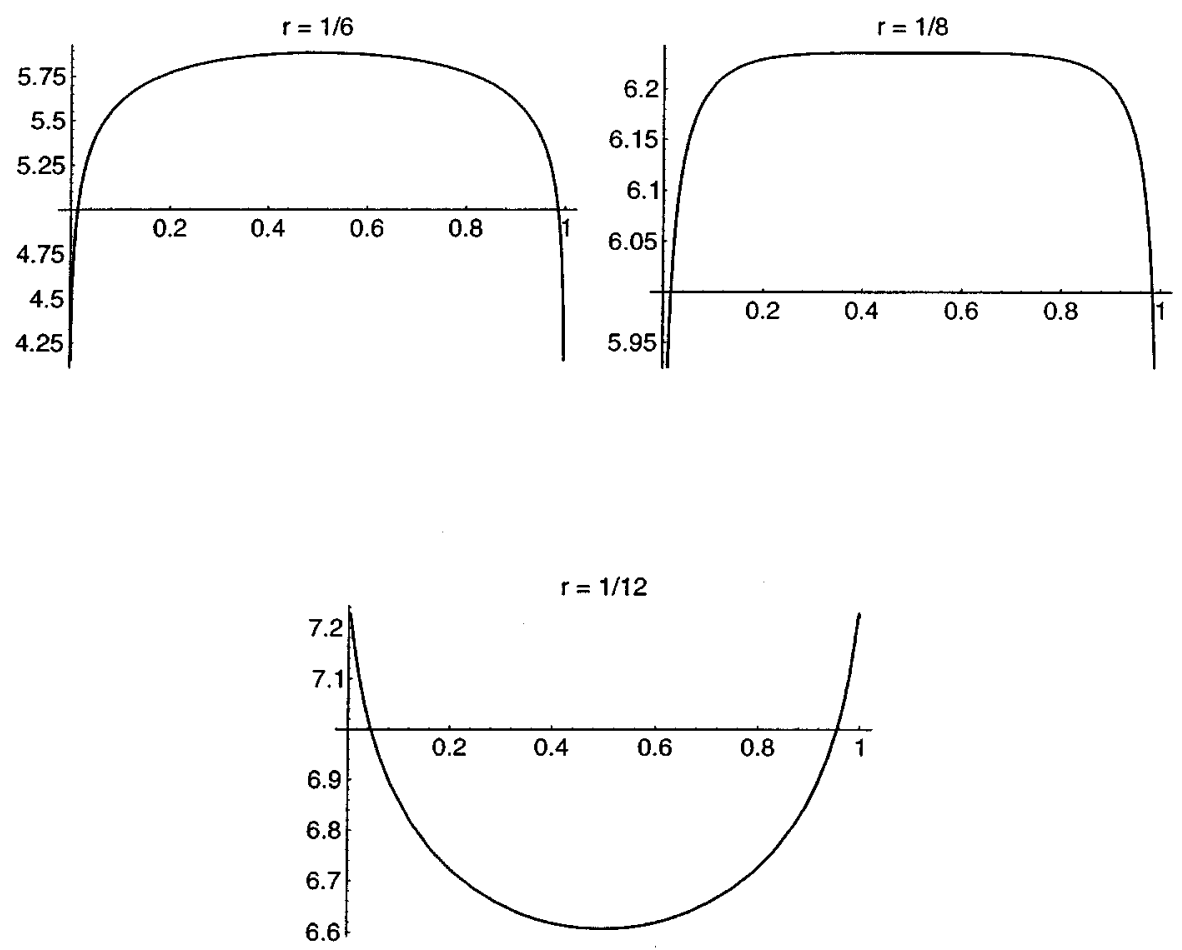

FiguRE 4. Graphs of $S_{r}$ for $r=1 / 6, r=1 / 8$, and $r=1 / 12$

Now properties (i), (ii), (iii) of $J_{1}$ and $J_{2}$ follow immediately from Lemma 1 and Lemma 2 since $J_{1}(x)=S_{1 / 12}(x)$ and $J_{2}(x)=T_{1 / 3,1 / 12}(x)$. Notice that $J_{0}(x)=$ $S_{1 / 6}(x)$. Hence, using Lemma 1 and the observation that

$$
\begin{aligned}
I\left(\frac{1}{2}\right) & =\int_{0}^{1} \frac{d t}{t^{1 / 2}(1-t)^{1 / 2}\left(1-\frac{1}{2} t\right)^{1 / 2}} \\
& =\left(\frac{1}{2}\right)^{1 / 2} \int_{0}^{1} \frac{d s}{s^{3 / 4}(1-s)^{1 / 2}} \quad(t=1-\sqrt{s}) \\
& =\left(\frac{1}{2}\right)^{1 / 2} B\left(\frac{1}{4}, \frac{1}{2}\right),
\end{aligned}
$$

we have $M_{4,0}=2^{7 / 6} B\left(\frac{1}{4}, \frac{1}{2}\right)$ as shown previously in [2].

Before giving the proofs of Lemma 1 and Lemma 2, we would like to make two remarks. First of all, the function $S_{1 / 8}$ has the "flattest" graph among the functions $S_{r}$, as a comparison of $S_{1 / 6}, S_{1 / 8}, S_{1 / 12}$ in Figure 4 clearly illustrates; indeed, it is so flat that

$$
I(x)+I(1-x) \approx \frac{2^{1 / 4} B\left(\frac{1}{4}, \frac{1}{2}\right)}{x^{1 / 8}(1-x)^{1 / 8}}
$$

for $x \in\left(\frac{1}{4}, \frac{3}{4}\right)$. Secondly, the local minimum of $J_{1}(x)\left(=S_{1 / 12}(x)\right)$ at $x=\frac{1}{2}$ actually corresponds to the form $X Y\left(X^{2}+Y^{2}\right)$ whose graph could be considered the "most 
symmetric" among quartic forms $F(X, Y)$ in the class $k=1$; hence, unlike the class $k=0$, the "natural" candidate $X Y\left(X^{2}+Y^{2}\right)$ does not maximize the quantity $\left|D_{F}\right|^{1 / 12} A_{F}$ when $k=1$.

We now proceed to prove Lemmas 1 and 2. As might be expected, both proofs make essential use of properties of the hypergeometric function.

Proof of Lemma 1. For each $r>0$, put

$$
P_{r}(x)=x^{r}(1-x)^{r}, \quad x \in(0,1) .
$$

Then

$$
S_{r}(x)=P_{r}(x) I(x)+P_{r}(1-x) I(1-x), \quad x \in(0,1) .
$$

We first show that $S_{r}(x)$ approaches zero as $x$ approaches the endpoints of the interval $(0,1)$. For this purpose, put $\epsilon=\min (r / 2,1 / 2)>0$. Then, using the estimates $1-x \leq 1-x t$ and $1-t \leq 1-x t$, we have

$$
\begin{aligned}
P_{r}(x) I(x) & \leq x^{r}(1-x)^{r-\epsilon} \int_{0}^{1} \frac{(1-x t)^{\epsilon} d t}{t^{1 / 2}(1-t)^{1 / 2}(1-x t)^{1 / 2}} \\
& \leq x^{r}(1-x)^{r-\epsilon} \int_{0}^{1} \frac{d t}{t^{1 / 2}(1-t)^{1-\epsilon}} \\
& =x^{r}(1-x)^{r-\epsilon} B(1 / 2, \epsilon)
\end{aligned}
$$

and so $P_{r}(x) I(x) \rightarrow 0$ as $x \rightarrow 1$. Similarly, $P_{r}(x) I(x) \rightarrow 0$ as $x \rightarrow 0$. Hence $S_{r}(x) \rightarrow 0$ as $x \rightarrow 0$ or 1 as claimed.

Now put

$$
V_{r}(x)=\frac{x(1-x)}{r P_{r}(x) P_{r}(1-x)} S_{r}^{\prime}(x), \quad x \in(0,1) .
$$

We will show that $S_{r}$ behaves as asserted in the statement of Lemma 1 by examining the signs of $V_{r}$ and $V_{r}^{\prime}$. Our argument will make essential use of the following differential equations for $P_{r}$ and $I$ :

$$
\begin{gathered}
x(1-x) P_{r}^{\prime}(x)-r(1-2 x) P_{r}(x)=0, \\
x(1-x) I^{\prime \prime}(x)+(1-2 x) I^{\prime}(x)-\frac{1}{4} I(x)=0 .
\end{gathered}
$$

(The first of these is easily verified; the second is a special case of the hypergeometric equation (see [1, p. 562]).) Notice that the signs of $V_{r}$ and $S_{r}^{\prime}$ are identical for $x \in(0,1)$ and that $V_{r}\left(\frac{1}{2}\right)=S_{r}^{\prime}\left(\frac{1}{2}\right)=0$.

From (25) we have

$$
S_{r}^{\prime}(x)=P_{r}^{\prime}(x) I(x)+P_{r}(x) I^{\prime}(x)-P_{r}^{\prime}(1-x) I(1-x)-P_{r}(1-x) I^{\prime}(1-x)
$$

and so, using the equation (26) for $P_{r}$, we can write

$$
\begin{aligned}
V_{r}(x)= & (1-2 x)\left\{\frac{I(x)}{P_{r}(1-x)}+\frac{I(1-x)}{P_{r}(x)}\right\} \\
& +\frac{x(1-x)}{r}\left\{\frac{I^{\prime}(x)}{P_{r}(1-x)}-\frac{I^{\prime}(1-x)}{P_{r}(x)}\right\} .
\end{aligned}
$$


Differentiating the two expressions on the right-hand side of this equation and using the equation for $P_{r}$ again, we have

$$
\begin{aligned}
\frac{d}{d x}(1-2 x) & \left\{\frac{I(x)}{P_{r}(1-x)}+\frac{I(1-x)}{P_{r}(x)}\right\} \\
=-2 & \left\{\frac{I(x)}{P_{r}(1-x)}+\frac{I(1-x)}{P_{r}(x)}\right\}+(1-2 x)\left\{\frac{I^{\prime}(x)}{P_{r}(1-x)}-\frac{I^{\prime}(1-x)}{P_{r}(x)}\right\} \\
& -\frac{r(1-2 x)^{2}}{x(1-x)}\left\{\frac{I(x)}{P_{r}(1-x)}+\frac{I(1-x)}{P_{r}(x)}\right\}
\end{aligned}
$$

and

$$
\begin{aligned}
\frac{d}{d x} \frac{x(1-x)}{r} & \left\{\frac{I^{\prime}(x)}{P_{r}(1-x)}-\frac{I^{\prime}(1-x)}{P_{r}(x)}\right\} \\
=\frac{1-2 x}{r} & \left\{\frac{I^{\prime}(x)}{P_{r}(1-x)}-\frac{I^{\prime}(1-x)}{P_{r}(x)}\right\} \\
+ & \frac{x(1-x)}{r}\left\{\frac{I^{\prime \prime}(x)}{P_{r}(1-x)}+\frac{I^{\prime \prime}(1-x)}{P_{r}(x)}\right\} \\
& -(1-2 x)\left\{\frac{I^{\prime}(x)}{P_{r}(1-x)}-\frac{I^{\prime}(1-x)}{P_{r}(x)}\right\} .
\end{aligned}
$$

Further, using the equation (27) for $I(x)$ we have

$$
\begin{aligned}
& \frac{x(1-x)}{r}\left\{\frac{I^{\prime \prime}(x)}{P_{r}(1-x)}+\frac{I^{\prime \prime}(1-x)}{P_{r}(x)}\right\}+\frac{1-2 x}{r}\left\{\frac{I^{\prime}(x)}{P_{r}(1-x)}-\frac{I^{\prime}(1-x)}{P_{r}(x)}\right\} \\
& \quad=\frac{1}{4 r}\left\{\frac{I(x)}{P_{r}(1-x)}+\frac{I(1-x)}{P_{r}(x)}\right\} .
\end{aligned}
$$

Hence, combining these three equations we find that

$$
V_{r}^{\prime}(x)=Q_{r}(x)\left\{\frac{I(x)}{P_{r}(1-x)}+\frac{I(1-x)}{P_{r}(x)}\right\}
$$

where

$$
Q_{r}(x)=\frac{1}{4 r}-2-\frac{r(1-2 x)^{2}}{x(1-x)} .
$$

Notice that the signs of $V_{r}^{\prime}$ and $Q_{r}$ are identical.

Now suppose that $r \geq 1 / 8$. Then $Q_{r}(x)<0$ for all $x$ (except possibly $x=\frac{1}{2}$ ), and so $V_{r}^{\prime}(x)<0$ for all such $x$, as well. Hence $V_{r}$ is decreasing. Since $V_{r}\left(\frac{1}{2}\right)=0$, it follows that $V_{r}(x)>0$ if $x \in\left(0, \frac{1}{2}\right)$ and $V_{r}(x)<0$ if $x \in\left(\frac{1}{2}, 1\right)$. Consequently,

$$
\begin{cases}S_{r}^{\prime}(x)>0 & \text { if } x \in\left(0, \frac{1}{2}\right) \\ S_{r}^{\prime}(x)<0 & \text { if } x \in\left(\frac{1}{2}, 1\right)\end{cases}
$$

since $V_{r}$ and $S_{r}^{\prime}$ have the same signs, and it follows that $x=\frac{1}{2}$ is the global maximum point for $S_{r}$. In fact, the point $x=\frac{1}{2}$ is the only critical point in this case.

On the other hand, suppose that $r<1 / 8$. Then $Q_{r}\left(\frac{1}{2}\right)>0$ and so $V_{r}^{\prime}\left(\frac{1}{2}\right)>0$. Hence $S_{r}^{\prime \prime}\left(\frac{1}{2}\right)>0$ and it follows that the point $x=\frac{1}{2}$ is a local minimum point for $S_{r}$. Since $S_{r}$ tends to zero at the endpoints of the interval $(0,1)$, the function $S_{r}$ must therefore have at least three critical points in $(0,1)$. We claim that there are 
exactly three critical points. Notice that the sign of $Q_{r}(x)$ is the same as the sign of the quadratic

$$
\left(\frac{1}{4 r}-2\right) \cdot x(1-x)-r(1-2 x)^{2}
$$

for $x \in(0,1)$ since $x(1-x)>0$, and that this quadratic has two roots in the interval $(0,1)$ which are symmetrically located with respect to the midpoint $\frac{1}{2}$. Letting $x_{0}$ denote the smaller root, we have

$$
\begin{cases}Q_{r}(x)<0 & \text { if } x \in\left(0, x_{0}\right), \\ Q_{r}(x)>0 & \text { if } x \in\left(x_{0}, 1-x_{0}\right), \\ Q_{r}(x)<0 & \text { if } x \in\left(1-x_{0}, 1\right) .\end{cases}
$$

Now, arguing as in the previous case, we find that the function $S_{r}$ has exactly one critical point in the interval $\left(x_{0}, 1-x_{0}\right)$. In fact,

$$
\begin{cases}S_{r}^{\prime}(x)<0 & \text { if } x \in\left(x_{0}, \frac{1}{2}\right), \\ S_{r}^{\prime}(x)>0 & \text { if } x \in\left(\frac{1}{2}, 1-x_{0}\right) .\end{cases}
$$

Hence, let us consider the interval $\left(0, x_{0}\right)$. Notice that $V_{r}$ is decreasing on this interval since $Q_{r}(x)<0$ - and consequently $V_{r}^{\prime}(x)<0$ also - for $x<x_{0}$. Notice further that

$$
V_{r}(x)=r^{-1} x^{1-2 r}(1-x)^{1-2 r} S_{r}^{\prime}(x)
$$

and that the quantity $r^{-1} x^{1-2 r}(1-x)^{1-2 r}$ is increasing for $x \in\left(0, \frac{1}{2}\right)$. Thus $S_{r}^{\prime}$ is decreasing on the interval $\left(0, x_{0}\right)$ and it follows that $S_{r}$ can have at most one critical point in this interval. Since $S_{r}$ tends to zero as $x$ approaches zero, we see that there is exactly one critical point. By symmetry, there is exactly one critical point in the interval $\left(1-x_{0}, 1\right)$. Therefore, we conclude that if $r<1 / 8$, then the function $S_{r}$ has exactly three critical points in the interval $(0,1)$ and, moreover, the point $x=\frac{1}{2}$ is a local minimum point.

This completes the proof of Lemma 1.

Proof of Lemma 2. The proof of Lemma 2 is similar to the proof of Lemma 1. However, here we will use the series expansion for $I(x)$ in addition to the differential equation (27). In particular, we will use the fact that

$$
I(x)=\pi \sum_{n=0}^{\infty}\left(\frac{\left(\frac{1}{2}\right)_{n}}{n !}\right)^{2} x^{n}
$$

where

$$
\left(\frac{1}{2}\right)_{n}=\left(\frac{1}{2}\right)\left(\frac{3}{2}\right)\left(\frac{5}{2}\right) \cdots\left(\frac{2 n-1}{2}\right) .
$$

(This follows from the definition of a hypergeometric function and its series representation [1], and can be derived directly from (7) of $\S 2$ by expanding $(1-x z)^{1 / 2}$ in a Taylor series and integrating term-by-term.)

To begin, note that $T_{r, s}(x)$ approaches zero as $x$ approaches either endpoint of the interval $(0,1)$. Indeed, arguing as in the proof of Lemma 1, we have

$$
x^{r}(1-x)^{s} I(x) \leq x^{r}(1-x)^{s-\epsilon} B(1 / 2, \epsilon)
$$


which tends to zero as $x \rightarrow 0$ or 1 (provided that $0<\epsilon<\min (s, 1 / 2)$ ). Hence $T_{r, s}$ has at least one maximum point in $(0,1)$. We will show that $T_{r, s}$ has at most one critical point in this interval, and Lemma 2 will follow.

Put

$$
V_{r, s}(x)=x^{1-r}(1-x)^{1-s} T_{r, s}^{\prime}(x) .
$$

Then from the definition of $T_{r, s}$,

$$
V_{r, s}(x)=r(1-x) I(x)-s x I(x)+x(1-x) I^{\prime}(x) .
$$

Differentiating $V_{r, s}$ and using the fact that

$$
\frac{d}{d x}\left(x(1-x) I^{\prime}(x)\right)=\frac{1}{4} I(x)
$$

(just another way to write the hypergeometric differential equation (27)), we find that

$$
V_{r, s}^{\prime}(x)=\left(\frac{1}{4}-r-s\right) I(x)+(r-x(r+s)) I^{\prime}(x) .
$$

Hence $V_{r, s}^{\prime}(x)<0$ if and only if

$$
(r-x(r+s)) I^{\prime}(x)<\left(r+s-\frac{1}{4}\right) I(x) .
$$

From the series definition of $I(x)$, we have

$$
I^{\prime}(x)=\pi \sum_{n=0}^{\infty}(n+1)\left(\frac{\left(\frac{1}{2}\right)_{n+1}}{(n+1) !}\right)^{2} x^{n}
$$

from which it follows (after some calculation) that

$$
(r-x(r+s)) I^{\prime}(x)=\pi \cdot \frac{r}{4}+\pi \sum_{n=1}^{\infty}\left(\frac{\left(\frac{1}{2}\right)_{n}}{n !}\right)^{2}\left(\frac{r}{n+1}\left(\frac{2 n+1}{2}\right)^{2}-(r+s) n\right) x^{n} .
$$

Similarly,

$$
\left(r+s-\frac{1}{4}\right) I(x)=\pi\left(r+s-\frac{1}{4}\right)+\pi \sum_{n=1}^{\infty}\left(\frac{\left(\frac{1}{2}\right)_{n}}{n !}\right)^{2}\left(r+s-\frac{1}{4}\right) x^{n} .
$$

Hence (29) will hold if each term in the latter series dominates the corresponding term in the former series, i.e.,

$$
\frac{r}{4}<r+s-\frac{1}{4}
$$

and

$$
\frac{r}{n+1}\left(\frac{2 n+1}{2}\right)^{2}-(r+s) n<r+s-\frac{1}{4}, \quad \text { for } n=1,2, \ldots
$$


Both of these conditions are satisfied if $3 r+4 s>1$ (the assumed condition of Lemma 2). Consequently, $V_{r, s}^{\prime}(x)<0$ for all $x \in(0,1)$.

Now $V_{r, s}^{\prime}(x)<0$ means that $V_{r, s}(x)$ is strictly decreasing; consequently, $V_{r, s}(x)=$ 0 for at most one $x \in(0,1)$. Since $V_{r, s}(x)$ and $T_{r, s}^{\prime}(x)$ have the same sign on $(0,1)$ (see (28)), it follows that $T_{r, s}$ has at most one critical point. However, $T_{r, s}$ has at least one maximum point since $T_{r, s}(x)>0$ for $x \in(0,1)$ and since $T_{r, s}(x)$ tends to zero at the endpoints. Therefore, $T_{r, s}$ has exactly one critical point - a global maximum point - in the interval $(0,1)$.

This completes the proof of Lemma 2 .

\section{REFERENCES}

1. M. Abramowitz and I. Stegun, Handbook of Mathematical Functions, Dover, New York, 1966. MR 34:8606

2. M. A. Bean, Binary forms, hypergeometric functions, and the Schwarz-Christoffel mapping formula, Trans. Amer. Math. Soc. 347 (12) (1995), 4959-4983. MR 96c:11038

3. - An isoperimetric inequality for the area of plane regions defined by binary forms, Compositio Math. 92 (2) (1994), 115-131. MR 95i:11078

4. _ An isoperimetric inequality related to Thue's equation, Bull. Amer. Math. Soc. 31 (2) (1994), 204-207. MR 95b:11034

5. $\quad$, A note on the Thue inequality, Proc. Amer. Math. Soc. 123 (7) (1995), 1975-1979. MR 95i: 11079

6. B. W. Char et al. Maple V Library Reference Manual, Springer-Verlag, New York, 1991.

7. R. C. Gunning, Introduction to Holomorphic Functions of Several Variables, Wadsworth \& Brooks-Cole, 1990. MR 92b:32001a,b,c

8. K. Mahler, Zur Approximation algebraischer Zahlen III, Acta Math. 62 (1934), 91-166.

9. J. Mueller and W. M. Schmidt, Thue's equation and a conjecture of Siegel, Acta Math. 160 (1988), 207-247. MR 89g:11029

10. W. M. Schmidt, Diophantine approximations and Diophantine equations, Lecture Notes in Math., vol. 1467, Springer-Verlag, New York, 1991. MR 94f:11059

11. A. Thue, Über Annäherungswerte algebraischer Zahlen, J. Reine Angew. Math. 135 (1909), 284-305.

12. S. Wolfram, Mathematica: a system for doing mathematics by computer, 2nd ed., AddisonWesley, New York, 1991.

Department of Mathematics, University of Michigan, Ann Arbor, Michigan 48109

E-mail address: mbean@math.lsa.umich.edu 